\title{
A Maximum Throughput Channel Allocation Protocol in Multi-channel Multi-user Cognitive Radio Network
}

\author{
Shi Wang, B.T. Maharaj, A.S. Alfa
}

\begin{abstract}
Channel allocation protocol plays a crucial role in ensuring that cognitive radio technology achieves more efficient radio resource utilization. To evaluate and compare the performance of different channel allocation protocols accurately, we need to model the cognitive radio system using various channel allocation protocols under one identical framework. Building such a framework is challenging. In this paper, we propose a method named distribution probability matrix (DPM) to describe the allocation results under different allocation protocols and a queueing analysis framework that uses DPM method. A framework that is capable of analyzing every individual secondary user (SU) in the system is developed. We also propose a maximum throughput (MT) channel allocation protocol aimed at achieving optimal throughput. Then we compare the MT protocol and existing protocols with the same objective using our proposed framework. The numerical results show that the MT protocol outperforms the existing protocols. Performance comparisons of different SUs are carried out to compare the protocols comprehensively. The convenience using our analytical framework of modeling different allocation protocols and evaluating the overall performances of the protocols is revealed throughout the analysis.
\end{abstract}

Index Terms: Cognitive radio, channel allocation protocol, maximum throughput, queueing analysis, performance evaluation.

\section{Introduction}

Cognitive radio (CR) technology is emerging as the most important solution to the growing conflict between the scarcity of spectrum resources and the proliferation of communication demands [1][2]. Different from the fixed spectrum assignment policy, CR technology enables the unlicensed user to utilize the temporarily unused licensed spectrum for promising applications, such as the smart grid, vehicular networks and emergency networks. The spectrum regulatory agencies (e.g., OFCOM in UK, FCC in the US, ICASA in South Africa) have attempted to open portions of the spectrum for opportunistic spectrum access. The development of the computing performances of devices also provides great potential for the effective use of radio frequency with CR technology. Thus the optimization of radio resource allocation using CR technology has become a pertinent topic to

This work was supported by the Sentech Chair in BWMC and DTI THRIP program.

S. Wang and B.T Maharaj are with the Department of Electrical, Electronic and Computer Engineering of the University of Pretoria, Pretoria, South Africa. (email:ihsgnaw@gmail.com, sunil.maharaj@up.ac.za).

Attahiru S. Alfa is with the Department of Electrical and Computer Engineering, University of Manitoba, Winnipeg, Manitoba, Canada R3T 5V6 and Department of Electrical, Electronic and Computer Engineering of the University of Pretoria, Pretoria, South Africa, (email: attahiru.alfa@umanitoba.ca).

B.T Maharaj is the corresponding author. study [3], and in these research studies, performance evaluation and optimization problems are effectively analyzed and solved by queueing theory.

Queueing analysis of telecommunication systems [4] is well developed and has proven to be efficient. Some researchers make the queueing analysis applicable to cognitive radio networks (CRN). In [5], the data transmission process of a CR system can be described by a finite-state Markov process model. Then from a cross-layer perspective, the information transmitted in the CRN is modeled as a discrete form of packet, and a finite state Markov chain is used to describe the data transmission process using adaptive modulation and coding (AMC) in [6][7]. Performance measures such as average throughput and packet loss rate are derived. The performance of delay statistics can also be effectively analysed, as in [8], with queueing analysis.

Using similar queueing analysis, it can be investigated how different channel allocation protocols affect performance measures and how a channel allocation protocol should be designed in the CRN can be investigated. In [9], contention-based and contention-free medium access methods are used to allocate the channels. In [10], opportunistic spectrum scheduling is applied. To create a general framework to describe the channel allocation protocol and to evaluate the performance, the authors develop a distribution probability matrix (DPM) in [11].

Maximizing throughput is one of the most important objectives of channel allocation protocol in wireless communication systems [12][13]. In the CR system, there are many channel allocation protocols aimed at achieving optimal throughput for the secondary user (SU) system, such as opportunistic spectrum scheduling [10][14][15] and the maximum rate scheme [8]. In [16], the authors take a further step to take the primary user's activity into considerations. However the existing protocol has some limitations. First, the criterions of these channel allocation protocols are only based on the transmission capacity through primary user (PU) channels of each SU, regardless of the transmission demand of the SU. Second, most analysis is about one selected SU in the SU system and the behavior of other SUs is assumed to be independent and identical to the selected SU. The correlation among SUs is ignored and the performance evaluation of the allocation protocol is presented only by the selected SU rather than all SUs.

The contributions of this work are as follows: first, we introduce a new channel allocation protocol, named the maximum throughput (MT) protocol, to allocate multiple PU channels to multiple SUs. The target of the allocation protocol is to maximize the overall throughput of the SU system considering both 


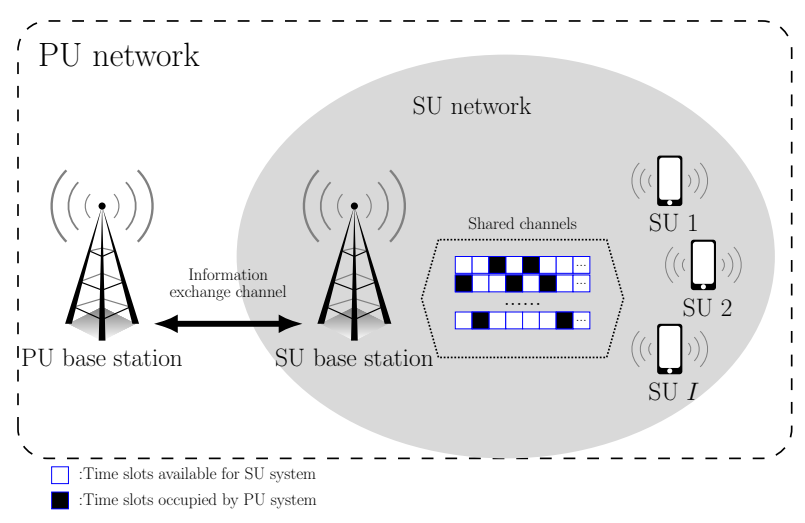

Fig. 1. System model.

the SU transmission capacities and the transmission demands. Then analysis of each individual SU in the system is carried out by applying the MT protocol in a queueing analysis framework. Numerical results show that MT protocol yields better throughput than the opportunistic spectrum scheduling in [8][10][14]. Moreover, using our framework, various overall performance metrics of the SU system can be obtained precisely and efficiently because all the SUs are modeled independently. Thus the study of the factors that affect optimal throughput is also enabled by our framework. Investigations on the impact of channel quality and SU traffic density on the throughput and delay are carried out and the discoveries are indicated by the numerical results.

The rest of the paper is organized as follows. We introduce the system model and assumptions about our system in Section II. Then we show the algorithm of our MT allocation protocol and how the queueing model is built in Section III. Numerical results and the discussions on them are shown in Section IV. Section V concludes the paper.

\section{The System Model and Assumptions}

\section{A. Basic Model Settings}

In this research, we consider the uplink channel allocation for an overlay [17] CRN with multiple secondary users and multiple channels. A reliable channel between the PU and SU system that can help exchange control information is assumed. The CRN comprises one SU base station and multiple SUs working with it within the coverage of one or more PU networks. The number of SUs is denoted as $M$. The number of PU channels that are available to the SU system is denoted as $J$. All the data in the system are in the form of discrete packets and the SU system is working under the same time slot structure that is synchronized with the PU system. Each SU has a finite buffer to store the packets waiting to be transmitted. The buffer size of the $i$-th SU is denoted by $K_{i}$. An example of the system is shown in Fig. 1.

The channel allocation protocol is the rule that the SU system uses to decide at the beginning of a time slot which SU can transmit on the channel where the PU is absent. The objective of the channel allocation protocol in CRN is to satisfy the quality of service requirements, such as maximizing the throughput, minimizing the packet loss rate, and minimizing the packet de- lay.

\section{B. Primary User Activity Model}

The SU system cannot use a channel while there is a PU transmitting on it. The interference caused by the PU activities on a channel are modeled as a time-homogeneous first-order Markov process with two states that are independent from the SU system [18]. Let $O^{j}(t) \in\{0,1\}$ represent the PU activity state of the $j$-th channel at the $t$-th time slot. When there is a PU transmitting, the state is called "busy" and $O^{j}(t)=0$, otherwise the state is called "free" and $O^{j}(t)=1$. The transition matrix of the process can be described as:

$$
\boldsymbol{P}_{\mathrm{PU}}^{j}=\left(\begin{array}{cc}
p_{\mathrm{b} \rightarrow \mathrm{b}}^{j} & 1-p_{\mathrm{b} \rightarrow \mathrm{b}}^{j} \\
1-p_{\mathrm{f} \rightarrow \mathrm{f}}^{j} & p_{\mathrm{f} \rightarrow \mathrm{f}}^{j}
\end{array}\right),
$$

where $p_{\mathrm{b} \rightarrow \mathrm{b}}^{j}=\operatorname{Pr}\left\{O^{j}(t)=0 \mid O^{j}(t-1)=0\right\}$ denotes the probability that there is a PU transmitting on the $j$-th channel (the "busy" state), given that there was a PU transmitting on the channel (the "busy" state) at the previous time slot, and $p_{\mathrm{f} \rightarrow \mathrm{f}}^{j}=$ $\operatorname{Pr}\left\{O^{j}(t)=1 \mid O^{j}(t-1)=1\right\}$ denotes the probability that the $j$-th channel state changes from "free" to "free". From our model, average PU occupancy duration $t_{\mathrm{PU}}$ and percentage of PU occupancy $\theta$ can be obtained as follows:

$$
\begin{gathered}
t_{\mathrm{PU}}=\frac{1}{1-p_{\mathrm{b} \rightarrow \mathrm{b}}^{j}} \\
\theta=\frac{1-p_{\mathrm{f} \rightarrow \mathrm{f}}^{j}}{2-p_{\mathrm{b} \rightarrow \mathrm{b}}^{j}-p_{\mathrm{f} \rightarrow \mathrm{f}}^{j}} .
\end{gathered}
$$

In our model, the PU occupancy state of all $J$ channels at the $t$-th time slot is represented by $\boldsymbol{o}$ :

$$
\boldsymbol{o} \in \mathcal{O}=\left\{\left(O^{1}(t), O^{2}(t), \ldots, O^{J}(t)\right) \mid O^{j}(t) \in\{0,1\}\right\} .
$$

\section{Secondary System Transmission Model}

The data transmission of the SU system is made under a time slot structure synchronized with the PU system. A time slot is divided into three consecutive parts, namely the spectrum sensing part, channel allocation part and data transmission part.

\section{C.1 Spectrum Sensing}

The SU system keeps sensing the existence of the PU transmission on every PU channel. In our model perfect spectrum sensing is assumed. At the end of the sensing part, the PU system estimates whether there is a PU transmitting on each channel and the transmission conditions (e.g: the signal-to-noise ratio at transceivers) of each SU mobile station.

\section{C.2 Channel Allocation}

According to the information the SU system collects in the spectrum sensing part, SU system allocates the available channels to the SUs for data transmission during the current time slot. In this paper, we assume that one PU channel can be allocated to only one SU. One SU can transmit through multiple PU channels. A flexible and configurable protocol DPM developed 


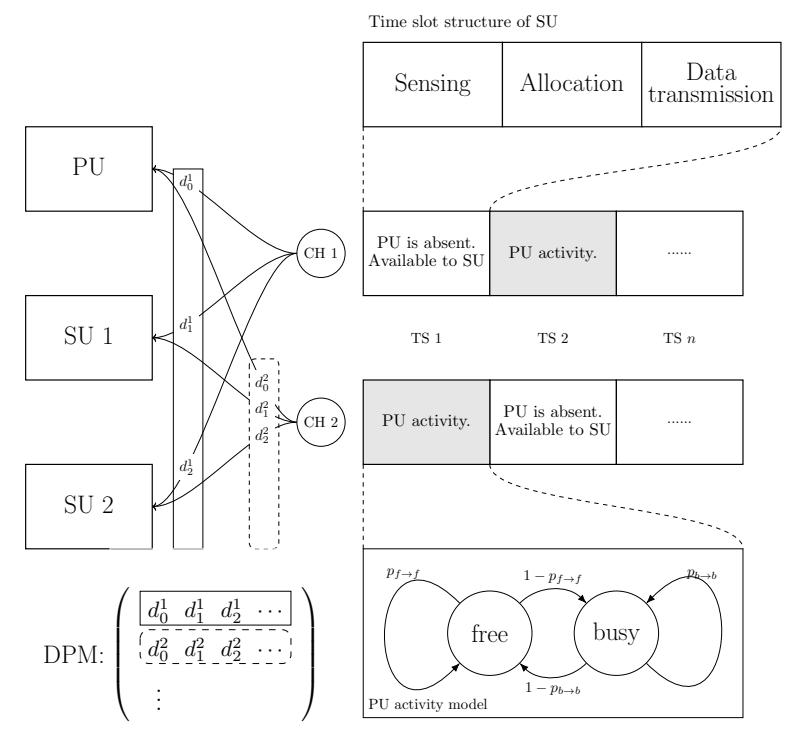

Fig. 2. Illustration of DPM, time slot structure of SU system and the PU activity model.

by the authors in [11] is used. It works as follows: We set up a $J$-by- $(M+1)$ distribution probability matrix:

$$
\boldsymbol{P}_{\mathrm{DPM}}=\left(\begin{array}{cccc}
d_{0}^{1} & d_{1}^{1} & \cdots & d_{M}^{1} \\
\vdots & \vdots & \ddots & \vdots \\
d_{0}^{J} & d_{1}^{J} & \cdots & d_{M}^{J}
\end{array}\right)
$$

where $d_{0}^{j}$ represents the probability that the $j$-th channel is not allocated to any SU, and $d_{i}^{j}$ for $i \neq 0$ represents the probability that the SU system allocates the $j$-th channel to the $i$-th SU. According to our assumptions, in $\boldsymbol{P}_{\mathrm{DPM}}: \sum_{i=0}^{M} d_{i}^{j}=1$ for any $j \in[1, J]$.

\section{C.3 Data Transmission}

The channel condition is modeled as a Markov process. Perfect control information between the PU and SU system is assumed. AMC is used in the SU system. The SNR of the $j$-th PU channel at the SU receiver is divided into $N_{\mathrm{SNR}}^{j}$ states. Let $c^{j}(t) \in\left\{1,2, \cdots, N_{\mathrm{SNR}}^{j}\right\}$ represent the channel condition state of the $j$-th channel at the $t$-th time slot. The evolution of the $j$-th channel condition states are modeled as a Markov chain using the same idea in [19]. The model is described by a $N_{\mathrm{SNR}}^{j}$ by- $N_{\mathrm{SNR}}^{j}$ transition matrix $\boldsymbol{P}_{\mathrm{CS}}^{j}$. According to the modulation method, we can obtain the data transmission rate in every channel state. Details are shown in Appendix A.

As the error recovery method, a stop-and-wait automatic request and repeat (ARQ) is applied. The average packet error rate is Per.

After data transmission, all packets arriving during the time slot will enter the SU buffer. If the total number of packets exceeds the buffer size, the overflow packets will be rejected.
III. The Queueing Analysis and Channel Allocation Protocol

\section{A. Queueing Model}

\section{A.1 Arrival Process}

We assume that the number of arrival packets at each SU during one time slot follows a Batch Bernoulli process [20]. Let us denote the probability that $j$ packets arrive during one time slot at the $i$-th SU by $\alpha_{i}(j)$. Hence we can use a probability vector $\boldsymbol{\alpha}_{i}$ to describe the process as follows:

$$
\boldsymbol{\alpha}_{i}=\left(\alpha_{i}(0), \alpha_{i}(1), \cdots, \alpha_{i}\left(v_{i}\right)\right),
$$

where $v_{i}$ is the maximum number of packets that can arrive at the $i$-th SU during one time slot and $\sum_{j=0}^{v_{i}} \alpha_{i}(j)=1$.

\section{A.2 State Space}

The state of the system can be described by the number of packets in the buffer of each SU and the channel states. The number of packets in the buffer of each SU is denoted by:

$$
\boldsymbol{b} \in \mathcal{B}=\left\{\left(b_{1}, b_{2}, \cdots, b_{M}\right) \mid b_{i} \in\left\{0,1, \cdots, K_{i}\right\}, i \in[1, M]\right\},
$$

where $b_{i}$ denotes the number of packets in the buffer of the $i$-th SU. The channel state is composed of all $J$ channel condition states to all $M$ SUs and the PU occupancy state of all $J$ channels. Here we assume that the SNR of every PU channel is identical to one SU. Thus the channel condition state of $J$ channels to $M$ SUs is denoted by:

$$
\boldsymbol{c} \in \mathcal{C}=\left\{\left(c_{1}, c_{2}, \cdots, c_{M}\right) \mid c_{i} \in\left[1, N_{\mathrm{SNR}}(i)\right]\right\},
$$

where $c_{i}$ is the channel condition states of the PU channels, and $N_{\mathrm{SNR}}(i)$ is the number of channel condition states of the $i$-th SU. Thus together with the PU occupancy state in eq. (4), the channel state of the system can be described by $\{\boldsymbol{o}, \boldsymbol{c}\}$, and the number of channel states is $N_{\mathrm{CS}}=2^{J} \cdot \prod_{i=1}^{M} N_{\mathrm{SNR}}(i)$.

The state space of the system can be denoted by:

$$
\Phi \triangleq\{(\boldsymbol{b}, \boldsymbol{o}, \boldsymbol{c}) \mid \boldsymbol{b} \in \mathcal{B}, \boldsymbol{o} \in \mathcal{O}, \boldsymbol{c} \in \mathcal{C}\} .
$$

The transition matrix between all the channel states $\{\boldsymbol{o}, \boldsymbol{c}\}$ is denoted by matrix $\boldsymbol{B}$ and $\boldsymbol{B}$ is obtained as:

$$
\boldsymbol{B}=\left(\boldsymbol{P}_{\mathrm{PU}}^{1} \otimes \cdots \otimes \boldsymbol{P}_{\mathrm{PU}}^{J}\right) \otimes \boldsymbol{P}_{\mathrm{CS}, 1} \otimes \cdots \otimes \boldsymbol{P}_{\mathrm{CS}, \mathrm{M}},
$$

where $\boldsymbol{P}_{\mathrm{CS}, \mathrm{i}}$ is the channel condition state transition probability matrix of the $i$-th SU in Appendix A and $\otimes$ is the Kronecker product.

\section{B. The Channel Allocation Protocol}

\section{B.1 Basic Ideas}

Our maximum throughput protocol does not only focuses on the capacity of available PU channels, but also takes the transmission demands of SU into consideration. The channel is allocated to the SU that has the potential to transmit most packets in the following time slot among all SUs. The potential throughput of one SU is denoted by the minimum between the channel capacity according to the channel condition state and AMC (ignoring the effect of ARQ) and the transmission demands (number of packets in buffer). In order to minimize the probability 


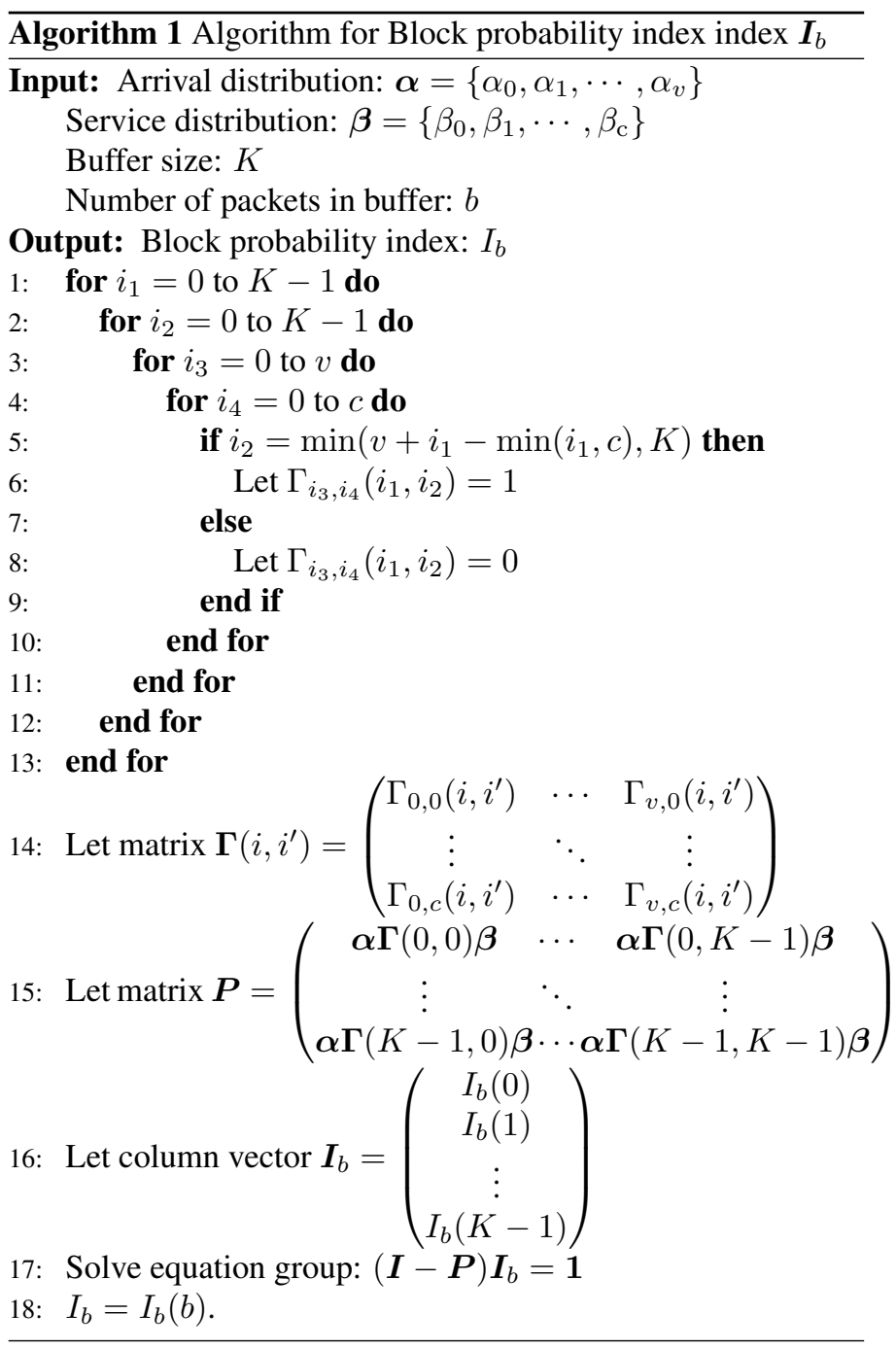

of rejecting arrival packet, when two or more SU have the same transmission potential, we will allocate the channel to the SU whose buffer is more likely to be full afterwards. We set up a block probability index (BPI) to describe how close the state of an SU's buffer is to the "full" state. Finally, the allocation results are represented in the form of DPM.

\section{B.2 Calculation of the Potential Throughput}

In our $M$-SU $J$-channel CRN, the allocation results are denoted by a vector $A=\left(a_{1}, a_{2}, \cdots, a_{J}\right)$, where $a_{j}=0$ represents that the $j$-th channel is not allocated to the SU system and $a_{j}=i, i \in\{1,2, \cdots, M\}$ represents that the $j$-th channel is allocated to the $i$-th SU. Given the channel condition state and the modulation schemes, we can obtain the capacity of the $j$-th channel to the $i$-th $\mathrm{SU}$, which is denoted by cap $\mathrm{p}_{\mathrm{i}}^{\mathrm{j}}$. Thus we can obtain the total capacity of all the channels that are allocated to the $i$-th $\mathrm{SU}$, which is denoted by cap $\mathrm{p}_{\mathrm{i}}$ as follows.

$$
\operatorname{cap}_{i}=\sum_{j \in\left\{j \mid a_{j}=i\right\}} \operatorname{cap}_{i}^{j} .
$$

The potential throughput can be denoted as $\mathrm{Thr}_{\mathrm{i}}=$ $\min \left(\mathrm{b}_{\mathrm{i}}, \operatorname{cap}_{\mathrm{i}}\right)$, where $b_{i}$ is the number of packets in the $i$-th SU's

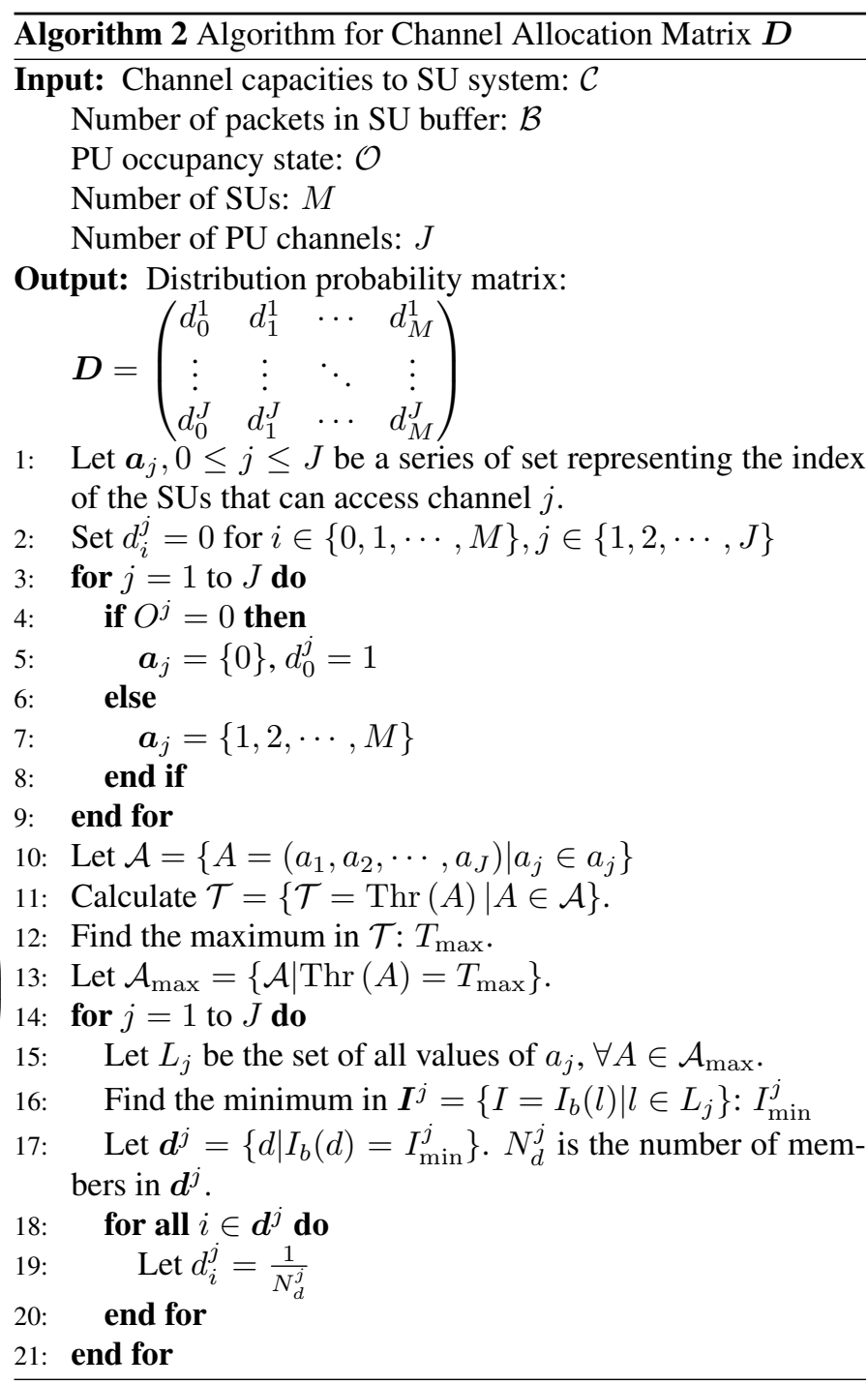

buffer. Finally we obtain the potential throughput of the given allocation result $A$ as:

$$
\operatorname{Thr}(A)=\sum_{i=1}^{M} \operatorname{Thr}_{\mathrm{i}} .
$$

\section{B.3 The Block Probability Index}

The BPI is defined as the average first passage time from the current state to the full buffer state, ignoring the PU activity. We use a $\mathrm{GeO}^{\mathrm{X}} / \mathrm{GeO}^{\mathrm{Y}} / 1 / \mathrm{K}$ queueing model to calculate the BPI. The inputs of the algorithm are the distribution of the number of arrival packets, the distribution of the number of packets that can be transmitted, the size of the buffer and the current number of packets in the buffer. The detail of the calculation is shown in Algorithm 1.

\section{B.4 Algorithm of the Allocation Protocol}

From the PU occupancy state: $\boldsymbol{o}$, we can list all possible channel allocation results. Thus, given the capacities of PU 
Table 1. Comparison of the runtime complexity of different channel allocation algorithms.

\begin{tabular}{|c|c|c|}
\hline Protocols & Best case & Worst case \\
\hline MT & $O\left(J \cdot \max \left(M^{2}, K^{2} v c, K^{4}\right)\right)$ & $O\left(M \cdot J \cdot \max \left(M^{J}, K^{2} v c, K^{4}\right)\right)$ \\
\hline MR & $O\left(M^{2} \cdot J\right)$ & $O\left(M^{J+1} \cdot J\right)$ \\
\hline MT no Index & $O\left(M^{2} \cdot J\right)$ & $O\left(M^{J+1} \cdot J\right)$ \\
\hline
\end{tabular}

channels to the SU system $\left(\mathcal{C}=\left[\begin{array}{ccc}\operatorname{cap}_{1}^{1} & \cdots & \operatorname{cap}_{M}^{1} \\ \vdots & \ddots & \vdots \\ \operatorname{cap}_{1}^{\mathrm{J}} & \cdots & \text { cap }_{\mathrm{M}}^{\mathrm{J}}\end{array}\right]\right)$ and the number of packets in the SUs' buffers, we can calculate the potential throughput of each allocation result. Let $\mathcal{A}_{\text {max }}$ denote the set of allocation results that have the maximum potential throughput. Let $L_{i}$ denote all the possible allocation results of the $i$-th channel in the set $\mathcal{A}_{\text {max }}$. Then calculate the BPI of all SUs in the set $L_{i}$. The distribution of the number of packets that each SU can transmit is calculated as described below. Assume that all the PU channels are utilized by the SU under the current SNR. Then using the probability of the channel condition state and the AMC transmission rate in Appendix A, the distribution can be obtained. Finally, the SUs in the set $L_{i}$ that have the minimum BPI share the opportunity to access the $i$-th channel. Details are shown in Algorithm 2.

\section{B.5 The Complexity Analysis of the Algorithm}

The calculation of the potential throughput and the BPI in the proposed MT algorithm increases the runtime complexity of the algorithm compared to the algorithms that ignore them. For comparison, a maximum rate (MR) protocol is implemented using our framework, as shown in Appendix B. The MR protocol is based on the principle that a PU channel is allocated to those SUs having the maximum transmission rate through that channel. A modified MT protocol (MT no Index) in which the BPI is removed is also proposed to provide a reference to investigate the trade-offs between the runtime complexity and the performance in the channel allocation algorithm design. The algorithm is shown in Appendix C. The details of the comparison of the complexity are listed in Table 1 . The contribution of the the potential throughput and the BPI to the complexity is related to the SU buffer size $K$, the maximum arrivals $v$ and the number of channel states $c$. The effect is significant when the number of SUs $(M)$ and the number of PU channels $(J)$ are small. When $M$ and $J$ become large, they will be the determining factors of the complexity of all algorithms. The complexity of the algorithms increases when the number of available PU channels increases. The best case is when there is only one PU channel available and the worst case is when all $J$ channels are available. The runtime statistics and performance comparison are further discussed with numerical results in Section IV.

\section{The Transition Matrix}

To build the transition matrix, the distribution of the number of packets that can be transmitted needs to be derived first. According to the AMC scheme, we can obtain the distribution of transmitted packets of each SU through each channel given the channel condition state, $c$. The number of transmitted trials of the $i$-th SU through the $j$-th channel is denoted as $C_{i}^{j}$. Accord- ing to the ARQ settings, we can obtain the probability that $x$ packets can be transmitted as follows:

$$
\mu(x)= \begin{cases}\left(\begin{array}{c}
C_{i}^{j} \\
x
\end{array}\right)(1-\mathrm{Per})^{\mathrm{x}} & \text { for } 0 \leq x \leq C_{i}^{j} \\
\operatorname{Per}^{C_{\mathrm{i}}^{\mathrm{j}}} & \text { for } x=0\end{cases}
$$

where $\left(\begin{array}{c}C_{i}^{j} \\ x\end{array}\right)$ is $C_{i}^{j}$ choose $x$. Given the allocation results: $\boldsymbol{A}=$ $\left(a_{1}, \cdots, a_{J}\right)$, we can obtain the distribution of the number of packets that can be transmitted by the $i$-th SU through the $j$-th channel:

$$
\boldsymbol{\mu}_{i}^{j}= \begin{cases}\left(\mu_{i}^{j}(0), \ldots, \mu_{i}^{j}\left(C_{i}^{j}\right)\right) & \text { for } a_{j}=i \\ (0) & \text { otherwise }\end{cases}
$$

Then the distribution of the number of packets that can be transmitted by the $i$-th SU given the channel state $\{\boldsymbol{o}, \boldsymbol{c}\}$ can be obtained as follows:

$$
\boldsymbol{\mu}_{i}(\boldsymbol{A}, \boldsymbol{o}, \boldsymbol{c})=\boldsymbol{\mu}_{i}^{1} * \boldsymbol{\mu}_{i}^{2} * \cdots * \boldsymbol{\mu}_{i}^{J},
$$

where $*$ is the convolution. The transition matrix that describes that the number of packets in the $i$-th SU's buffer changes from $b_{i}$ to $b_{i}^{\prime}$ under $\boldsymbol{A}$ :

$$
\boldsymbol{P}\left(b_{i}, b_{i}^{\prime}, \boldsymbol{A}\right)=\left[\begin{array}{ccc}
\boldsymbol{\alpha}_{i} \boldsymbol{\Gamma}(0,0) \boldsymbol{\mu}_{i}(\boldsymbol{A}) & \cdots & \boldsymbol{\alpha}_{i} \boldsymbol{\Gamma}(0, K) \boldsymbol{\mu}_{i}(\boldsymbol{A}) \\
\vdots & \ddots & \vdots \\
\boldsymbol{\alpha}_{i} \boldsymbol{\Gamma}(K, 0) \boldsymbol{\mu}_{i}(\boldsymbol{A}) & \cdots & \boldsymbol{\alpha}_{i} \boldsymbol{\Gamma}(K, K) \boldsymbol{\mu}_{i}(\boldsymbol{A})
\end{array}\right]
$$

where $\Gamma\left(i, i^{\prime}\right)$ is an auxiliary matrix, the elements of the matrix are derived as follows:

$$
\Gamma_{v, c}\left(i, i^{\prime}\right)=\left\{\begin{array}{ll}
1 & \text { when } i^{\prime}=\min (v+i-\min (i, c), K) \\
0 & \text { otherwise }
\end{array} .\right.
$$

Let vector $\boldsymbol{b}=\left(b_{1}, b_{2}, \cdots, b_{M}\right)$ denote the number of packets in each SU's buffer at the current time slot, and $\boldsymbol{b}^{\prime}=$ $\left(b_{1}^{\prime}, b_{2}^{\prime}, \cdots, b_{M}^{\prime}\right)$ denote the number of packets at the next time slot. Then we can get a coefficient matrix given $\boldsymbol{b}, \boldsymbol{b}^{\prime}$ and $\boldsymbol{A}$ :

$\boldsymbol{T}\left(\boldsymbol{b}, \boldsymbol{b}^{\prime}, \boldsymbol{A}\right)=\boldsymbol{P}\left(b_{1}, b_{1}^{\prime}, \boldsymbol{A}\right) \otimes \boldsymbol{P}\left(b_{2}, b_{2}^{\prime}, \boldsymbol{A}\right) \otimes \cdots \otimes \boldsymbol{P}\left(b_{M}, b_{M}^{\prime}, \boldsymbol{A}\right)$.

Using Algorithm 2, we can obtain a DPM $D$ given a system state $\phi \in \Phi$, and using the elements in $\boldsymbol{D}$ we can obtain:

$$
\boldsymbol{T}_{\boldsymbol{b}, \boldsymbol{b}^{\prime}}(\phi)=\sum_{\mathrm{A} \in \mathcal{A}}\left(\prod_{j=1}^{J} d_{a_{j}}^{j} \boldsymbol{T}\left(\boldsymbol{b}, \boldsymbol{b}^{\prime}, \boldsymbol{A}\right)\right)
$$

where $d_{i}^{j}$ is from the DPM $D$ and $\mathcal{A}$ is the set of all possible allocation results. We list all the coefficients according to the 
index of channel condition states, and extend it into a matrix with the same dimensions as $\boldsymbol{B}$ :

$$
\boldsymbol{T}_{\boldsymbol{b}, \boldsymbol{b}^{\prime}}=\left[\begin{array}{c}
\boldsymbol{T}_{\boldsymbol{b}, \boldsymbol{b}^{\prime}}(1) \\
\vdots \\
\boldsymbol{T}_{\boldsymbol{b}, \boldsymbol{b}^{\prime}}(\phi) \\
\vdots \\
\boldsymbol{T}_{\boldsymbol{b}, \boldsymbol{b}^{\prime}}\left(N_{\mathrm{CS}}\right)
\end{array}\right] \otimes \mathbf{1}_{1 \times\left(\prod_{i=1}^{M}\left(K_{i}+1\right) \cdot N_{\mathrm{CS}}\right)} .
$$

To enumerate all coefficient matrices, we map $\beta_{\text {index }}$ to $\boldsymbol{b}=$ $\left(b_{1}, b_{2}, \cdots, b_{M}\right)$ using the following equation:

$$
\text { index }=\sum_{i=1}^{M} b_{i} \times \text { base }_{i-1}
$$

where base ${ }_{i}=\prod_{j=i}^{M}\left(K_{i}+1\right)$.

The transition matrix is divided into $\prod_{i=1}^{M}\left(K_{i}+1\right) \times$ $\prod_{i=1}^{M}\left(K_{i}+1\right)$ blocks and obtained as follows:

$$
\boldsymbol{P}=\left[\begin{array}{ccc}
\boldsymbol{T}_{\beta_{0}, \beta_{0}} \circ \boldsymbol{B} & \ldots & \boldsymbol{T}_{\beta_{0}, \beta_{B}} \circ \boldsymbol{B} \\
\vdots & \ddots & \vdots \\
\boldsymbol{T}_{\beta_{B}, \beta_{0}} \circ \boldsymbol{B} & \ldots & \boldsymbol{T}_{\beta_{B}, \beta_{B}} \circ \boldsymbol{B}
\end{array}\right],
$$

where $\circ$ is the entrywise product.

\section{Performance Evaluation}

We can apply the QR algorithm to get the steady probability vector: $\boldsymbol{\pi}^{\prime}$ given the transition matrix $\boldsymbol{P} . \boldsymbol{\pi}^{\prime}$ is a 1 by $\prod_{i=1}^{M}\left(K_{i}+\right.$ 1) $\times N_{\mathrm{CS}}$ vector. According to the sequences of the states in $\boldsymbol{P}$, we can map a vector $(\boldsymbol{b}, \boldsymbol{o}, \boldsymbol{c})$ to any system state. The steady probability vector is reorganized in the form of $\pi(\boldsymbol{b}, \boldsymbol{o}, \boldsymbol{c})$.

\section{D.1 Queue Length Distribution}

Since we have obtained the steady probability vector of each system state, the distribution of the number of packets in the $i$ SU's buffer can be derived. Let $P\left(q_{i}=l\right)$ denote the probability that there are $l$ packets waiting to be transmitted in the $i$-th SU's buffer; this can be calculated as follows:

$$
P\left(q_{i}=l\right)=\sum_{\boldsymbol{b} \in B_{i}(l)} \boldsymbol{\pi}(\boldsymbol{b}, \boldsymbol{o}, \boldsymbol{c}),
$$

where $B_{i}(l)=\left\{\left(b_{1}, \cdots, b_{M}\right) \mid b_{i}=l, b_{j} \in\left[0, K_{j}\right], j \neq i\right\}$, which represents the set of the state of SU buffers when the $i$-th SU has $l$ packets in the buffer. The average queue length of the $i$-th SU can be obtained as:

$$
\overline{q_{i}}=\sum_{l=1}^{K_{i}} l \cdot P\left(q_{i}=l\right) .
$$

\section{D.2 Average Throughput}

The average throughput is described by the expected number of packets that can be transmitted during one time slot. First, we can calculate the distribution of the transmission the $i$-th SU can make under each system state $\phi=(\boldsymbol{b}, \boldsymbol{o}, \boldsymbol{c}) \in \Phi$, which is denoted by $\boldsymbol{t}_{i}(\boldsymbol{b}, \boldsymbol{o}, \boldsymbol{c})$ as follows:

$$
\boldsymbol{t}_{i}(\boldsymbol{b}, \boldsymbol{o}, \boldsymbol{c})=\sum_{\left.A \in \mathcal{A}^{\mid}(\rangle\right)} d_{i}^{j} \boldsymbol{\mu}_{i}(A, \boldsymbol{o}, \boldsymbol{c})
$$

where $d_{i}^{j}$ is from the DPM $\boldsymbol{D}$ obtained from $\phi$ and $\left.\mathcal{A}^{\mid}(\rangle\right)$is the set of allocation results when the $j$-th channel is allocated to the $i$-th SU. Then we can obtain the average throughput under $(\boldsymbol{b}, \boldsymbol{o}, \boldsymbol{c})$ :

$$
\operatorname{Thr}(\mathrm{b}, \mathrm{o}, \mathrm{c})=\sum_{\beta} \min \left(\beta, \mathrm{b}_{\mathrm{i}}\right) \cdot \mathrm{t}_{\mathrm{i}}(\mathrm{b}, \mathrm{o}, \mathrm{c}, \beta),
$$

where $t_{i}(\boldsymbol{b}, \boldsymbol{o}, \boldsymbol{c}, \beta)$ is the $(\beta+1)$-th element of $\boldsymbol{t}_{i}(\boldsymbol{b}, \boldsymbol{o}, \boldsymbol{c})$, which is the probability that $\beta$ transmissions have been made by the $i$-th SU under $(\boldsymbol{b}, \boldsymbol{o}, \boldsymbol{c})$. Thus the average throughput can be derived as:

$$
\overline{\operatorname{Thr}}=\sum_{\phi \in \Phi} \operatorname{Thr}(\mathrm{b}, \mathrm{o}, \mathrm{c}) \cdot \boldsymbol{\pi}(\mathrm{b}, \mathrm{o}, \mathrm{c})
$$

\section{D.3 Average Rejection}

The average rejection is the expected number of packets that are rejected at the beginning of one time slot. First we can calculate the average number of rejected under $(\boldsymbol{b}, \boldsymbol{o}, \boldsymbol{c})$ :

$$
\begin{array}{r}
\operatorname{Rej}(\mathrm{b}, \mathrm{o}, \mathrm{c})=\sum_{\mathrm{y}=0}^{\mathrm{v}_{\mathrm{i}}} \sum_{\beta} \max \left(0, b_{i}-\beta+y-K_{i}\right) \\
\cdot \min \left(\beta, b_{i}\right) \cdot t_{i}(\boldsymbol{b}, \boldsymbol{o}, \boldsymbol{c}, \beta) .
\end{array}
$$

Thus the average number of rejected packets can be derived as:

$$
\overline{\operatorname{Rej}}=\sum_{\phi \in \Phi} \operatorname{Rej}(\mathrm{b}, \mathrm{o}, \mathrm{c}) \cdot \boldsymbol{\pi}(\mathrm{b}, \mathrm{o}, \mathrm{c}) \text {. }
$$

\section{D.4 Average Delay}

We use the expected time to transmit all packets that are in the buffer at the beginning of a time slot to describe the delay performance. To calculate it, the state space is extended with another state $f$, which is the number of packets that have already been transmitted by a selected SU. The extended system state is $\Phi^{\prime} \triangleq\{\boldsymbol{b}, \boldsymbol{o}, \boldsymbol{c}, f\}$. Then we need to modify the transition matrix based on $\boldsymbol{P}$ given that there are $F$ packets in the selected SU's buffer. First, we build an extending band matrix given $\{\boldsymbol{b}, \boldsymbol{o}, \boldsymbol{c}\}$ and $\boldsymbol{t}_{i}$ obtained as in eq. (25):

$$
\boldsymbol{P}_{\mathrm{ex}}(\boldsymbol{b}, \boldsymbol{o}, \boldsymbol{c}, f)=\left[\begin{array}{ccc}
P_{0,0} & \cdots & P_{0, f} \\
\vdots & \ddots & \vdots \\
P_{f, 0} & \cdots & P_{f, f}
\end{array}\right]
$$

where

$P_{j, j^{\prime}}= \begin{cases}t_{i}\left(\boldsymbol{b}, \boldsymbol{o}, \boldsymbol{c}, j^{\prime}-j\right) & \text { for } j^{\prime}-j \in\left[0, \max \left(\boldsymbol{t}_{i}(\boldsymbol{b}, \boldsymbol{o}, \boldsymbol{c})\right)\right] \\ 0 & \text { otherwise }\end{cases}$

where $\max \left(\boldsymbol{t}_{i}(\boldsymbol{b}, \boldsymbol{o}, \boldsymbol{c})\right)$ is the maximum number of packets that can be transmitted under $(\boldsymbol{b}, \boldsymbol{o}, \boldsymbol{c})$. Then extend $\boldsymbol{P}$ row by row as follows:

$$
\boldsymbol{P}_{\text {delay }}^{\prime}(f)=\left[\begin{array}{c}
\boldsymbol{P}(\phi(0)) \otimes \boldsymbol{P}_{\mathrm{ex}}(\phi(0), f) \\
\vdots \\
\boldsymbol{P}\left(\phi\left(R_{m}\right)\right) \otimes \boldsymbol{P}_{\mathrm{ex}}\left(\phi\left(R_{m}\right), f\right)
\end{array}\right]
$$




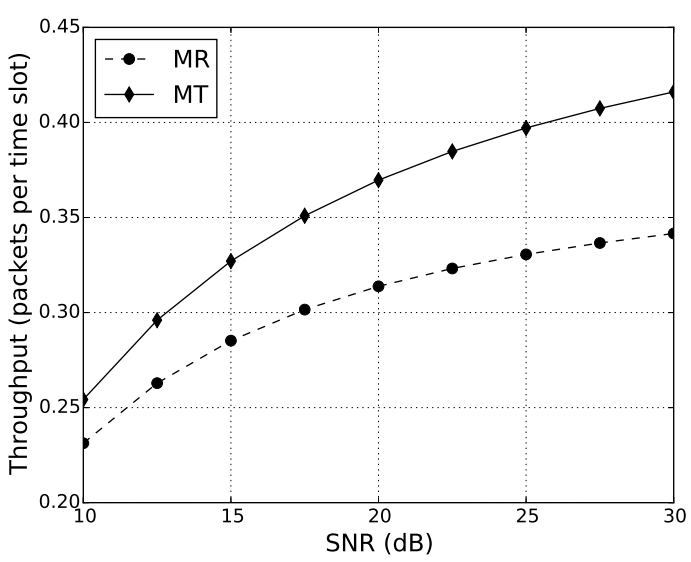

Fig. 3. Throughput versus SNR under MT protocol and MR protocol.

where $R_{m}=\prod_{i=1}^{M}\left(K_{i}+1\right) \cdot N_{\mathrm{CS}}$ is the size of $\boldsymbol{P}$, and $P(\phi(r))$ is the $r$-th row of matrix $\boldsymbol{P}$. The absorbing states are when all the packets in the buffer are transmitted $\left(b_{i}=0\right.$ or $\left.f=F\right)$, then remove them from $\boldsymbol{P}_{\text {delay }}^{\prime}$ to get $\boldsymbol{P}_{\text {delay }}$. The average first passage time to the absorbing states under each state is denoted as $\boldsymbol{S}$, which is a $\left(K_{s} \cdot \prod_{i \neq s}\left(K_{i}+1\right) \cdot N_{\mathrm{CS}}\right)$-by- 1 column vector $(s$ is the label of the selected SU). Then solve $\boldsymbol{S}$ from the following equations:

$$
\left(\boldsymbol{I}-\boldsymbol{P}_{\text {delay }}\right) \cdot \boldsymbol{S}=\mathbf{1}
$$

where $\boldsymbol{I}$ is the identity matrix, $\mathbf{1}$ is a column vector whose elements are all 1 and size is the same as $\boldsymbol{S}$. Let $\boldsymbol{S}(F)$ be the solution of $\boldsymbol{S}$ given $F$. Thus the average first passage time from the state of $F$ packets in the buffer to the empty buffer state is:

$$
d(F)=\sum_{\boldsymbol{b} \in B_{s}(F)} S(F, \boldsymbol{b}, \boldsymbol{o}, \boldsymbol{c}) \cdot \boldsymbol{\pi}(\boldsymbol{b}, \boldsymbol{o}, \boldsymbol{c}),
$$

where $B_{s}(F)$ is the set of all states of SU buffers when the selected SU's buffer has $F$ packets in it. Finally the average delay of the $i$-th SU can be obtained as:

$$
\overline{\mathrm{Dly}}=\sum_{t=1}^{K_{i}} d(t) .
$$

\section{Numerical Results and Discussion}

To control complexity without loss of generality, we implement a model with two users and two channels to show the performance of our protocol and the advantages of our analytical framework. The default settings are as follows:

- Channel condition: $P_{\text {target }}=0.01, f_{m}=10 \mathrm{~Hz}$

- PU activity model: $t_{P U}=1, \theta=50 \%$

- Arrival process: $\alpha_{1}=\alpha_{2}=\{0.5,0.5\}$

- SU buffer size: $K_{1}=6, K_{2}=6$

- ARQ setting: Per $=0.01$

\section{A. Performance Comparison}

In this part, we first compare the throughput of our protocol with the MR protocol under different SNRs. Fig. 3 shows the throughputs of the MT and MR protocols under different

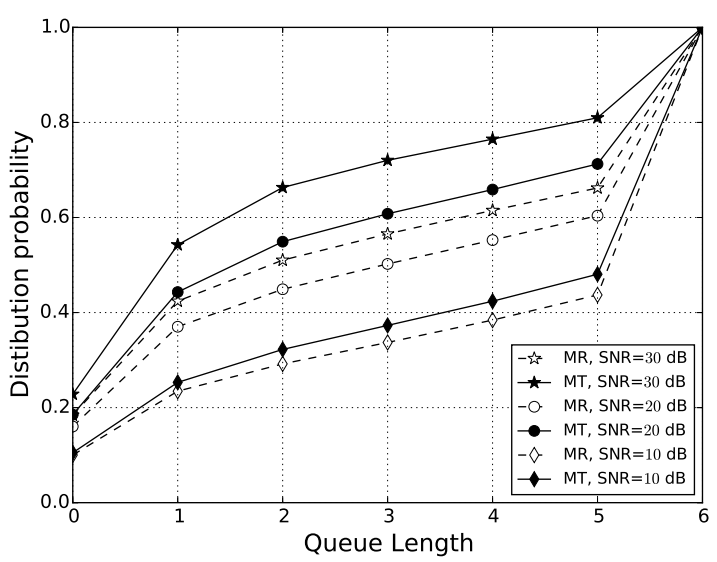

Fig. 4. Queue length distribution under MT protocol and MR protocol.

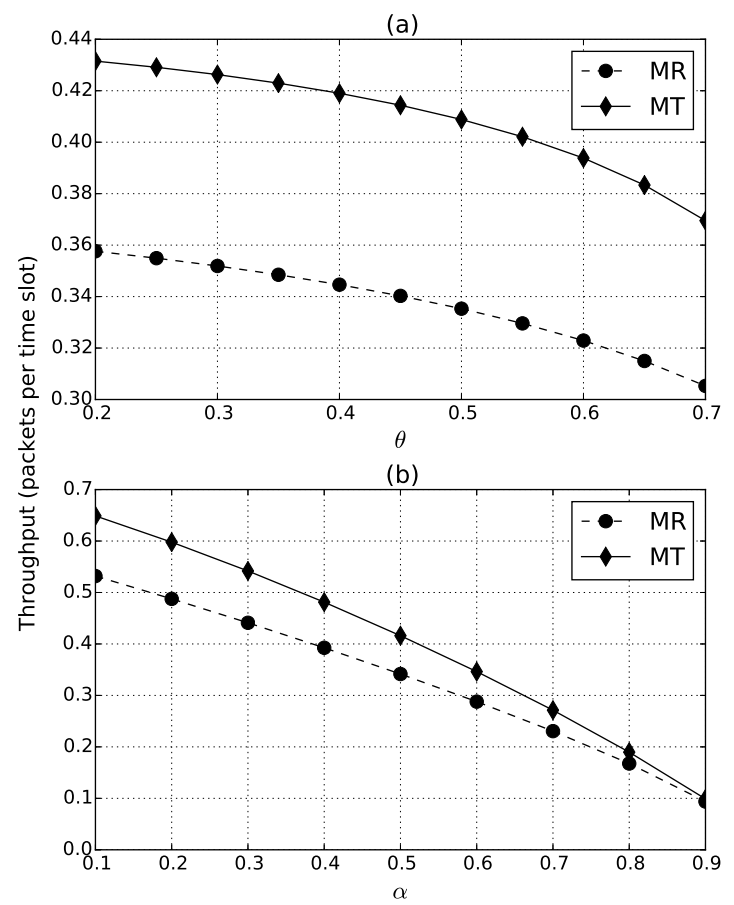

Fig. 5. Throughput improvements versus different system settings.

SNRs. Using our protocol, there is $10 \%$ to $20 \%$ improvement on throughput under the given conditions. The demand of buffer size can also be improved by our protocol. Fig. 4 shows the queue length distributions under MT and MR protocols versus different SNRs. Then we change the PU activity parameters and SU arrival process parameters to investigate how the other system parameters affect the throughput improvement. The results are shown in Fig. 5. In Fig. 5(a), $\theta$ is the PU occupancy probability of both channels; in Fig. 5(b), $\alpha$ is the probability that no packets arrived during the previous time slot. We can observe from the results that our protocol will achieve greater improvement when there is more PU activity or there are more SU arrival 


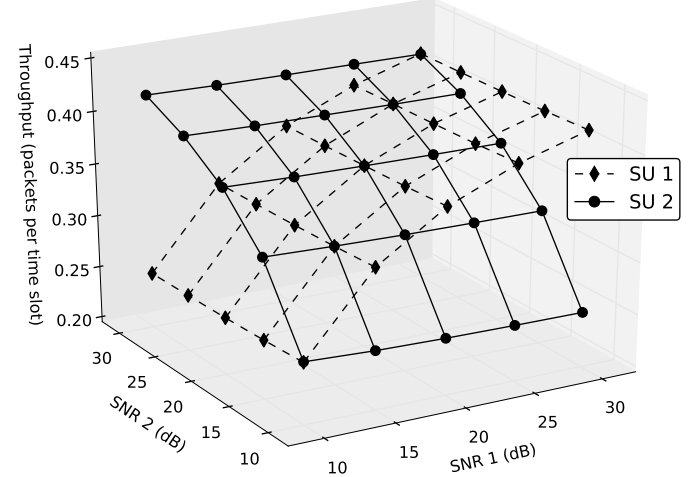

Fig. 6. Throughput of SU 1 and SU 2 versus SNRs.

packets. We can conclude that our protocol can outperform the MR protocol on optimizing throughput and it is able to achieve more improvement when the SU system has more spectrum resources or the traffic of the SU system is heavier.

\section{B. Performance Analysis of Multiple SUs}

One advantage of our analytical framework is that all PU channels can be set independently, and the performance of all SUs can be obtained simultaneously. We investigate the influence of channel allocation protocol on both SUs when the system parameters vary. Fig. 6 shows the throughput of SU 1 and SU 2 when the SNR of the two SUs vary. We choose an SNR setting to compare the performance between the MT and MR protocols in terms of the throughput of the two SUs. The results are shown in Fig. 7. We can observe a shortcoming of the MR protocol. When one of the SUs has a better transmission condition (higher SNR), the MR protocol inclines to allocate the channels to it even if it does not have enough transmission demands (packets waiting in the buffer), which will obviously waste the precious spectrum resources. Moreover, the increase of the SNR of the SU under worse transmission conditions does not affect the performance of the SU under better conditions. In Fig. 8, the average delay of SU 1 and SU 2 under different SNRs is shown and we find that the delay performance of the SU with better SNR remains the same while the other SU's SNR increases. Using our protocol, the increase of one SU's SNR does not affect another SU's transmission seriously when there is sufficient spectrum resource. This proves that our MT protocol can guarantee the transmission quality of each SU in the system, thus achieving higher resource utilization efficiency besides improving throughput.

The analyses above reveal the advantages of our framework. First, our framework is capable of modeling various channel allocation protocols and comparing their performance in different situations. Second, using our framework, the individual performances of the SUs can be obtained simultaneously and from these results we are able to investigate how a channel allocation protocol works in more detail.

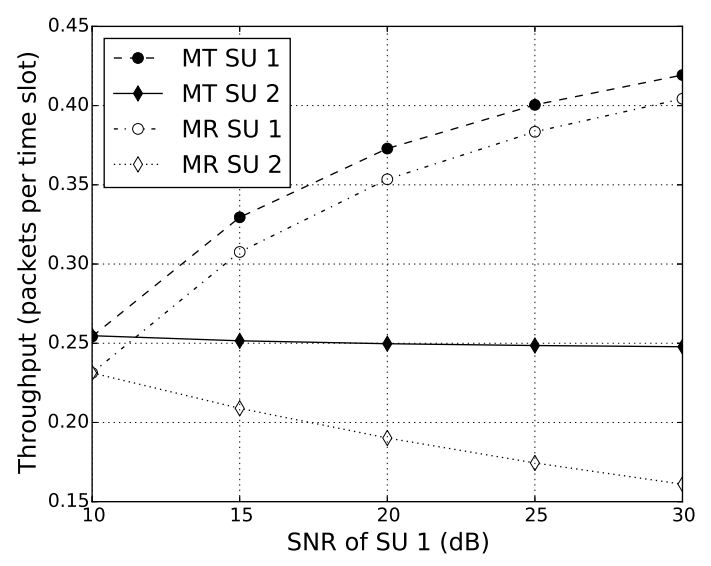

Fig. 7. Throughput of 2 SUs versus SNR of SU 1. The SNR of SU 2 is $10 \mathrm{~dB}$.

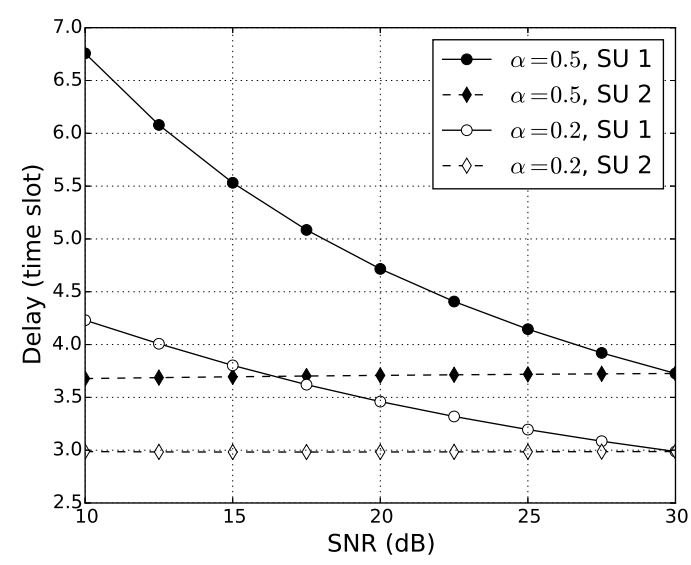

Fig. 8. Average delay of SU versus SNR. The SNR of SU 2 is $30 \mathrm{~dB}$. $\alpha$ is the probability that no packets arrived at SU 1 during the previous time slot.

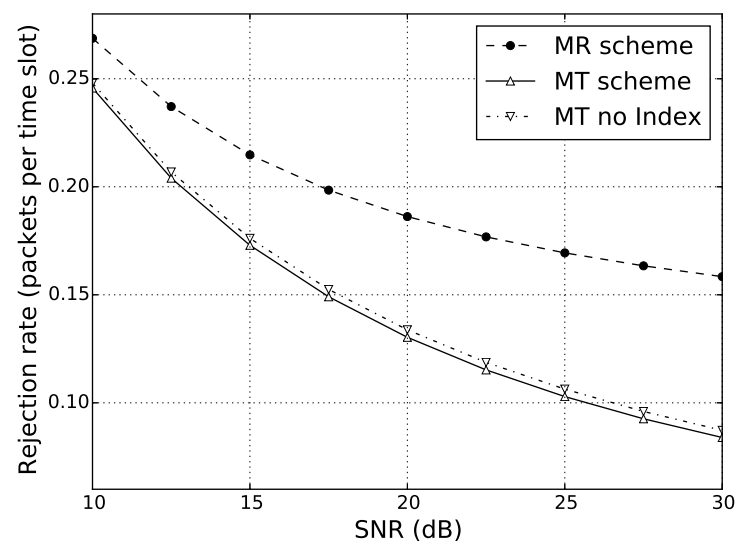

Fig. 9. Packet rejection rate versus SNR under different protocols.

\section{Discussion on Trade-offs between Performance and Com- plexity}

In this part, the performance and runtime complexity of the MT, MR and MT without BPI are compared through numerical 


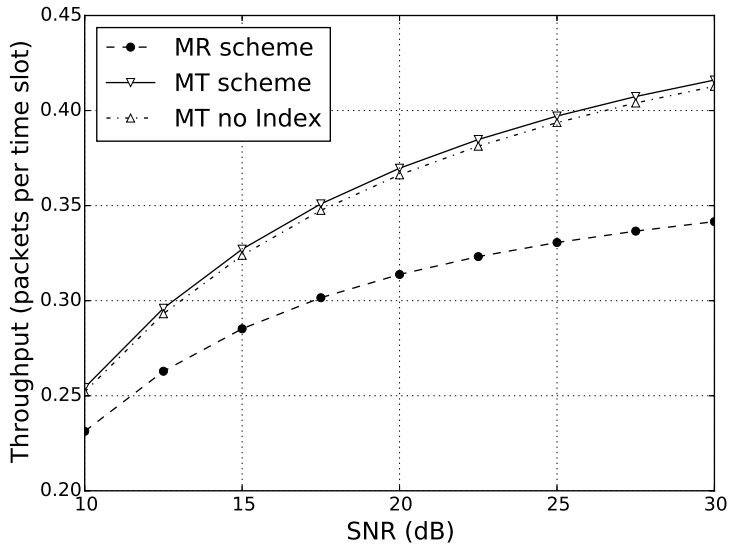

Fig. 10. Throughput versus SNR under different protocols.

results to illustrate the trade-offs in channel allocation protocol design. Because BPI is designed to improve the packet rejection rate, the rejection rate together with the throughput under different SNR settings are compared in Fig. 9 and Fig. 10. The results reveal that the potential throughput part provides most of the improvement on throughput and rejection rate and BPI can be applied to achieve utmost performance improvement. The codes of the three protocols are run in MATLAB 1000 times in an identical hardware environment; the runtime statistics are shown in Fig. 11. The potential throughput and the BPI part of the MT protocol are the reason it outperforms the MR. However, the trade-offs between the computing resource consumption and performance improvement should be considered in practice. For example, the BPI part may not be a good choice if the computing resources of the SU system are limited because this part consumes great computing resources but does not yield much improvement. This part of the analysis shows that our framework is able to reveal the trade-offs in channel allocation protocol design from some specific angles and provide a reference when designing and evaluating CR channel allocation protocols.

\section{Conclusions}

In this paper, we introduce an improved channel allocation protocol named the maximum throughput protocol. Compared to previous channel allocation protocols with similar ideas, such as MR, it can improve the SU system performance of throughput by $10 \%$ to $20 \%$ under the selected system settings. Performance measures such as the queue length in the buffer and the packet rejection rate are also improved. The negative effects of SUs under poor transmission conditions in the system on the performance of one SU, such as throughput and packet delay, can also be reduced by our protocol.

To carry out the analysis of different channel allocation protocols, we establish a queueing model using the concept of the DPM proposed by the authors and it proves to be practicable to describe channel allocation protocols in queueing analysis frameworks. Our framework is able to describe the behavior of each individual SU in the system independently and to evaluate various performance measures of each SU under various system and environment settings. This makes our framework capable

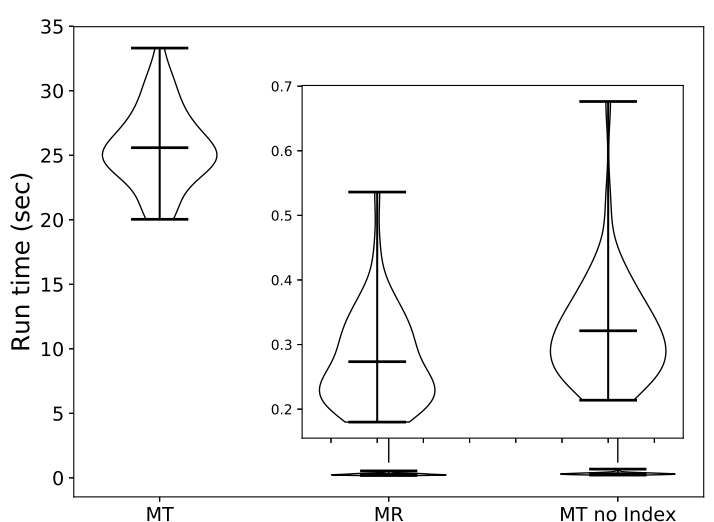

Fig. 11. Runtime statistics of different channel allocation protocols (1000 times).

of comparing different channel allocation protocols from more angles and in more detail. The ideas presented in this paper can also provide convenience in channel allocation design and performance optimization for CRN.

\section{APPENDICES}

\section{Channel state model}

To make better use of the limited channel resources, AMC scheme is applied by the SU system [21]. In this paper, the convolutionally coded $M_{n}$-ary rectangular and square QAM modes are used as the modulation method, which are adopted from the HIPERLAN/2 and IEEE 802.11a standards [22]. The details of the AMC scheme are listed in Table 2.

According to the SNR at the SU's transceiver, we divide the $j$-th channel condition into $N_{\mathrm{SNR}}^{j}$ states. Different modulation schemes are used to make the average packet error rates under each state all equal to a required packet error rate $P_{\text {target }}$. Thus, the boundary of these states in terms of SNR can be written in [6] as:

$$
\Gamma_{n}=\frac{1}{g_{n}} \ln \left(\frac{a_{n}}{P_{\text {target }}}\right),
$$

where $\Gamma_{n} \in\left\{\Gamma_{0}, \Gamma_{1}, \ldots, \Gamma_{N_{\mathrm{SNR}}^{j}}\right\}$ denotes the boundary SNR of each state. Then by setting $\Gamma_{0}=0$ and $\Gamma_{N_{\mathrm{SNR}}^{j}+1}=\infty$, we can obtain the $N_{\mathrm{SNR}}^{j}+1$ SNR boundary of the $N_{\mathrm{SNR}}^{j}$ states. When the SNR at an SU's receiver satisfies $\gamma \in\left(\Gamma_{0}, \Gamma_{1}\right)$, the SU does not transmit any packets because of the low SNR and when $\gamma \in\left(\Gamma_{n}, \Gamma_{n+1}\right), n \in\left\{1,2, \ldots, N_{\mathrm{SNR}}^{j}-1\right\}$, the $\mathrm{SU}$ is transmitting using mode $n$.

Then we can determine how the channel condition state evolves. We assume that the channel is Rayleigh: the SNR is exponentially distributed with the probability density function:

$$
p\left(\gamma_{i}^{j}\right)=\frac{1}{\bar{\gamma}_{i}^{j}} \exp \left(-\frac{\gamma_{i}^{j}}{\bar{\gamma}_{i}^{j}}\right), \quad \gamma_{i}^{j} \geq 0
$$

where $\gamma_{i}^{j}$ is the received SNR of the $i$-th SU on the $j$-th channel, 
Table 2. Transmission modes with convolutionally coded modulation

\begin{tabular}{c||c|c|c}
\hline & Mode 1 & Mode 2 & Mode 3 \\
\hline \hline Modulation & BPSK & QPSK & 8-QAM \\
\hline Coding rate $R_{c}$ & $1 / 2$ & $1 / 2$ & $3 / 4$ \\
\hline Rate(bits/symbol) & 0.5 & 1 & 1.5 \\
\hline$a_{n}$ & 274.7229 & 90.2514 & 67.6181 \\
\hline$g_{n}$ & 7.9932 & 3.4998 & 1.6883 \\
\hline$\Gamma_{n}(\mathrm{~dB})$ & -1.5331 & 1.0942 & 3.9722 \\
\hline
\end{tabular}

and $\bar{\gamma}_{i}^{j}$ is the average SNR. The steady state probability of the $k$-th state of the $i$-th SU on the $j$-th channel can be obtained by:

$$
\pi_{\mathrm{CS}, \mathrm{i}}^{j}(k)=\int_{\Gamma_{k}}^{\Gamma_{k+1}} p\left(\gamma_{i}^{j}\right) \mathrm{dx} .
$$

Let $\boldsymbol{P}_{\mathrm{CS}, \mathrm{i}}^{j}=\left(\begin{array}{ccc}p_{0,0} & \cdots & p_{0, N_{\mathrm{SNR}}^{j}-1} \\ \vdots & \ddots & \vdots \\ p_{N_{\mathrm{SNR}}^{j}-1,0} & \cdots & p_{N_{\mathrm{SNR}}^{j}-1, N_{\mathrm{SNR}}^{j}-1}\end{array}\right)$ denote the state transition probability matrix of the $i$-th SU on the $j$-th channel, where $p_{a, b}$ denotes the transition probability from state $a$ to state $b$. According to [23], the transition probabilities can be obtained by:

$$
\begin{gathered}
p_{k, k+1} \approx \frac{f_{m} T_{p}}{\pi_{\mathrm{CS}, \mathrm{i}}^{j}(k)} \sqrt{\frac{2 \pi \Gamma_{k+1}}{\bar{\gamma}_{i}^{j}}} \exp \left(-\frac{\Gamma_{k+1}}{\bar{\gamma}_{i}^{j}}\right), \\
p_{k, k-1} \approx \frac{f_{m} T_{p}}{\pi_{\mathrm{CS}, \mathrm{i}}^{j}(k)} \sqrt{\frac{2 \pi \Gamma_{k}}{\bar{\gamma}_{i}^{j}}} \exp \left(-\frac{\Gamma_{k}}{\bar{\gamma}_{i}^{j}}\right),
\end{gathered}
$$

where $f_{m}$ is the maximum Doppler frequency. We assume that the transition occurs only between adjacent states: $p_{j, k}=0$, for any $|j-k|>1$, and $p_{k, k}=1-p_{k, k+1}-p_{k, k-1}$, then with (35)(39), the transition probability matrix $\boldsymbol{P}_{\mathrm{CS}}^{j}$ can be obtained. Let $V_{\text {rate }}(k)$ represent the number of packets that can be transmitted in one time slot when the channel condition state is $k$. According to the rate in Table $2, V_{\text {rate }}(k), k \in\{0,1,2,3\}=\{0,1,2,3\}$.

\section{Algorithm for Maximum Rate Protocol}

The calculation of function $\mathrm{Thr}_{\max }$ is similar to the potential throughput in eq. (12). Given the channel condition state and the modulation schemes, we can obtain the total capacity of all the channels that are allocated to the $i$-th SU, which is denoted by cap $_{\mathrm{i}}$. The throughput of the given allocation result $A$ under MR is as:

$$
\operatorname{Thr}_{\mathrm{MR}}(\mathrm{A})=\sum_{\mathrm{i}=1}^{\mathrm{M}} \mathrm{cap}_{\mathrm{i}} .
$$

III. Algorithm for Maximum Throughput Protocol without BPI

\section{REFERENCES}

[1] J. Mitola III and G. Q. Maguire Jr, "Cognitive radio: making software radios more personal," Personal Communications, IEEE, vol. 6, no. 4, pp. 13-18, 1999 .

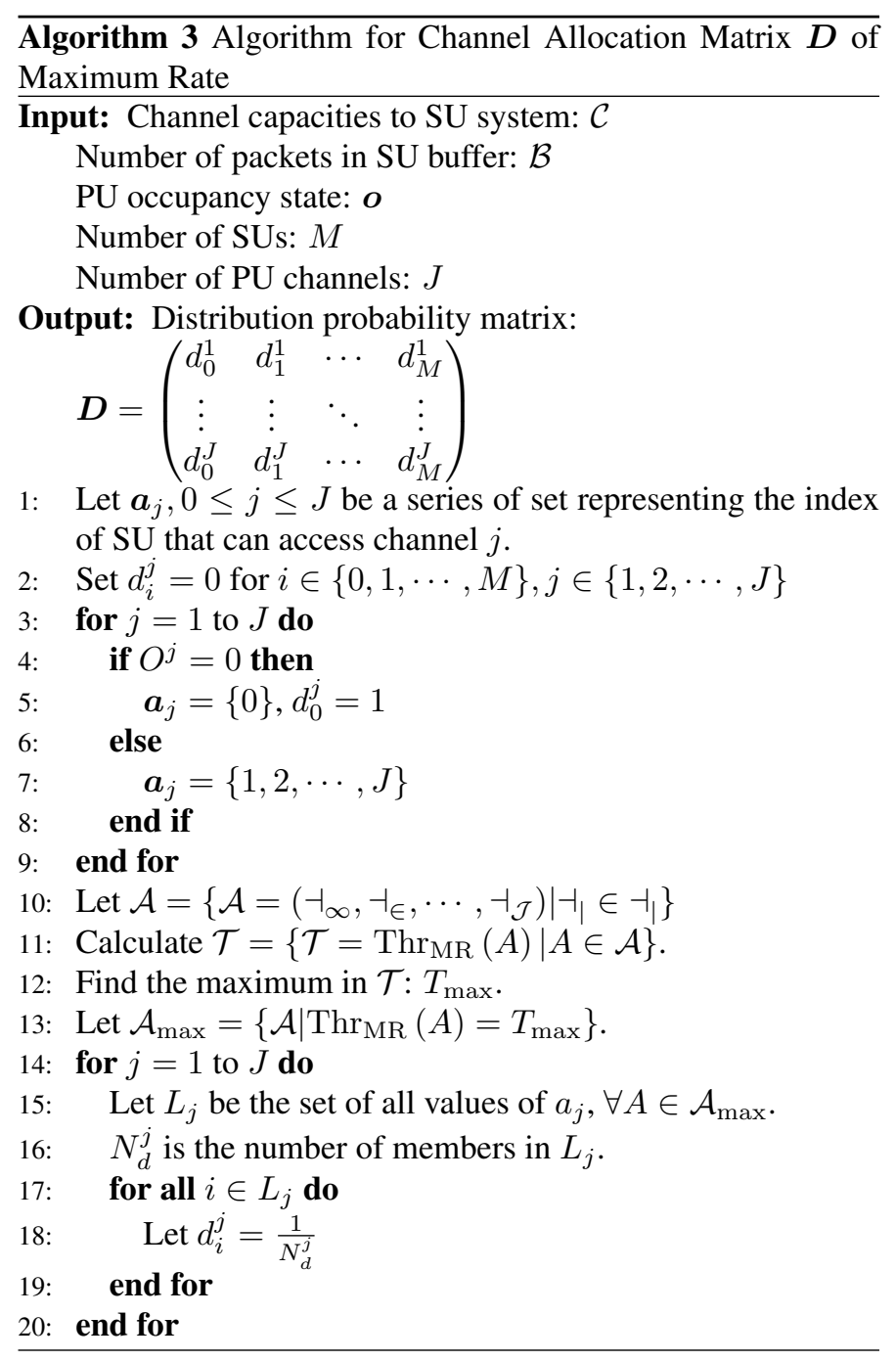

[2] S. Haykin, "Cognitive radio: Brain-empowered wireless communications," IEEE J. Sel. Areas Commun., vol. 23, no. 2, pp. 201-220, Feb. 2005.

[3] A. S. Alfa, B. T. Maharaj, S. Lall, and S. Pal, "Mixed-integer programming based techniques for resource allocation in underlay cognitive radio networks: a survey," Journal of Communications and Networks, vol. 18, no. 5, pp. 744-761, 2016.

[4] A. S. Alfa, Queueing theory for telecommunications: discrete time modelling of a single node system. Springer Science \& Business Media, 2010.

[5] P. Sadeghi, R. A. Kennedy, P. B. Rapajic, and R. Shams, "Finite- state Markov modeling of fading channels - a survey of principles and applications," IEEE Signal Processing Magazine, vol. 25, no. 5, pp. 57-80, 2008.

[6] Q. Liu, S. Zhou, and G. B. Giannakis, "Cross-layer combining of adaptive modulation and coding with truncated arq over wireless links," Wireless Communications, IEEE Transactions on, vol. 3, no. 5, pp. 1746-1755, 2004.

[7] Q. Liu, S. Zhou, and G. B. Giannakis, "Queuing with adaptive modulation and coding over wireless links: cross-layer analysis and design," Wireless Communications, IEEE Transactions on, vol. 4, no. 3, pp. 1142-1153, 2005.

[8] L. B. Le, E. Hossain, and A. S. Alfa, "Delay statistics and throughput performance for multi-rate wireless networks under multiuser diversity," IEEE Transactions on Wireless Communications, vol. 5, no. 11, pp. 3234 3243, 2006.

[9] J. Wang, A. Huang, L. Cai, and W. Wang, "On the queue dynamics of multiuser multichannel cognitive radio networks," Vehicular Technology, IEEE Transactions on, vol. 62, no. 3, pp. 1314-1328, 2013.

[10] M. M. Rashid, M. J. Hossain, E. Hossain, and V. K. Bhargava, "Opportunistic spectrum scheduling for multiuser cognitive radio: a queueing 
$\overline{\text { Algorithm } 4 \text { Algorithm for Channel Allocation Matrix } D \text { of }}$ Maximum Throughput without BPI

Input: Channel capacities to SU system: $\mathcal{C}$

Number of packets in SU buffer: $\mathcal{B}$

PU occupancy state: $\boldsymbol{o}$

Number of SUs: $M$

Number of PU channels: $J$

Output: Distribution probability matrix:

$$
\boldsymbol{D}=\left(\begin{array}{cccc}
d_{0}^{1} & d_{1}^{1} & \cdots & d_{M}^{1} \\
\vdots & \vdots & \ddots & \vdots \\
d_{0}^{J} & d_{1}^{J} & \cdots & d_{M}^{J}
\end{array}\right)
$$

1: Let $\boldsymbol{a}_{j}, 0 \leq j \leq J$ be a series of set representing the index of SU that can access channel $j$.

2: $\quad$ Set $d_{i}^{j}=0$ for $i \in\{0,1, \cdots, M\}, j \in\{1,2, \cdots, J\}$

3: for $j=1$ to $J$ do

4: $\quad$ if $O^{j}=0$ then

5: $\quad \boldsymbol{a}_{j}=\{0\}, d_{0}^{j}=1$

6: $\quad$ else

8

9:

10: $\quad$ Let $\mathcal{A}=\left\{\mathcal{A}=\left(\dashv_{\infty}, \dashv_{\in}, \cdots, \dashv_{\mathcal{J}}\right) \mid \dashv_{\mid} \in \dashv_{1}\right\}$

11: Calculate $\mathcal{T}=\{\mathcal{T}=\operatorname{Thr}(A) \mid A \in \mathcal{A}\}$.

12: Find the maximum in $\mathcal{T}: T_{\max }$.

13: Let $\mathcal{A}_{\max }=\left\{\mathcal{A} \mid \operatorname{Thr}(A)=T_{\max }\right\}$.

14: for $j=1$ to $J$ do

15: Let $L_{j}$ be the set of all values of $a_{j}, \forall A \in \mathcal{A}_{\max }$.

16: $\quad N_{d}^{j}$ is the number of members in $L^{j}$.

17: $\quad$ for all $i \in L^{j}$ do

18: $\quad$ Let $d_{i}^{j}=\frac{1}{N_{d}^{j}}$

19: end for

20: end for

analysis," Wireless Communications, IEEE Transactions on, vol. 8, no. 10, pp. 5259-5269, 2009.

[11] S. Wang, B. T. Maharaj, and A. S. Alfa, "Resource allocation and performance measures in multi-user multi-channel cognitive radio networks," In Vehicular Technology Conference (VTC Spring), 2016 IEEE.

[12] M. Tao, Y.-C. Liang, and F. Zhang, "Resource allocation for delay differentiated traffic in multiuser OFDM systems", IEEE Transactions on Wireless Communications, vol. 7, no. 6, 2008

[13] X. Wang and G. B. Giannakis, "Resource allocation for wireless multiuser OFDM networks," IEEE Transactions on Information theory, vol. 57, no. 7, pp. 4359-4372, 2011.

[14] Y. Wang, Y. Xu, L. Shen, C. Xu, and Y. Cheng, “Two-dimensional pomdpbased opportunistic spectrum access in time-varying environ- ment with fading channels," Journal of Communications and Networks, vol. 16, no. 2, pp. 217-226, 2014.

[15] F. Zhou, N. C. Beaulieu, J. Cheng, Z. Chu, and Y. Wang, "Robust maxmin fairness resource allocation in sensing-based wideband cognitive radio with SWIPT: imperfect channel sensing," IEEE Systems Journal, 2017

[16] J. Lee and H.-K. Park, "Channel prediction-based channel allocation scheme for multichannel cognitive radio networks," Journal of Communications and Networks, vol. 16, no. 2, pp. 209-216, 2014.

[17] I. F. Akyildiz, W.-Y. Lee, M. C. Vuran, and S. Mohanty, "Next generation/dynamic spectrum access/cognitive radio wireless networks: a survey," Computer Networks, vol. 50, no. 13, pp. 2127-2159, 2006.

[18] S. Senthuran, A. Anpalagan, H. Y. Kong, A. Karmokar, and O. Das, "An opportunistic channel access scheme for interweave cognitive radio systems," Journal of Communications and Networks, vol. 16, no. 1, pp. 5666, 2014.

[19] Q. Zhang, S. Kassam et al., "Finite-state Markov model for Rayleigh fading channels," Communications, IEEE Transactions on, vol. 47, no. 11, pp. 1688-1692, 1999.
[20] T. G. Robertazzi, Computer networks and systems: queueing theory and performance evaluation. Springer Science \& Business Media, 2012.

[21] A. J. Goldsmith and S.-G. Chua, "Adaptive coded modulation for fading channels," Communications, IEEE Transactions on, vol. 46, no. 5, pp. 595-602, 1998.

[22] A. Doufexi, S. Armour, M. Butler, A. Nix, D. Bull, J. McGeehan, and P. Karlsson, "A comparison of the HIPERLAN/2 and IEEE 802.11 a wireless LAN standards," Communications Magazine, IEEE, vol. 40, no. 5, pp. 172-180, 2002.

[23] H. S. Wang and N. Moayeri, "Finite-state Markov channel - a useful model for radio communication channels," Vehicular Technology, IEEE Transactions on, vol. 44, no. 1, pp. 163-171, 1995.

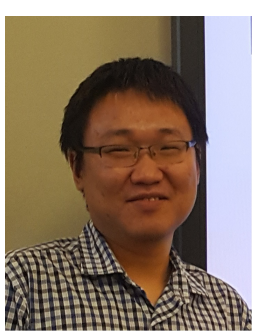

Shi Wang received B.Eng and M.Eng degree from Beijing University of Telecommunication, Beijing on 2006 and 2009, respectively. He is currently a Ph.D. student in the Department of Electrical, Electronic and Computer Engineering of the University of Pretoria, Pretoria, South Africa. His research focus includes cognitive radio networks, radio resource allocation in wireless networks and queueing theory.

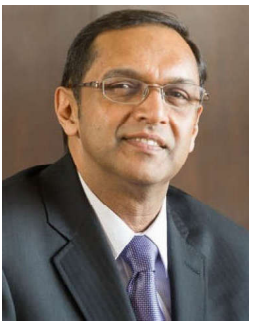

B. T. Maharaj Sunil Maharaj received his PhD in Engineering the area of Wireless Communications from the University of Pretoria. Dr Maharaj is a full professor and currently holds the research position of Sentech Chair in Broadband Wireless Multimedia Communications in the Department of Electrical, Electronic and Computer Engineering at the University of Pretoria. His research interests are in OFDM-MIMO systems, massive MIMO, cognitive radio resource allocation and 5G Cognitive Radio Sensor Networks.

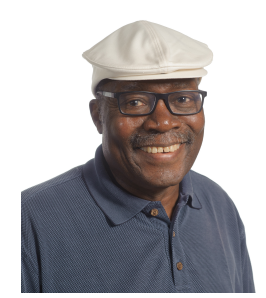

Attahiru S. Alfa is Professor Emeritus at the University of Manitoba, Department of Electrical and Computer Engineering and also a UP/CSIR co-hosted SARChI Chair professor at the University of Pretoria, Department of Electrical, Electronic and Computer Engineering. His research covers, but not limited to, the following areas: queueing theory and applications, optimization, performance analysis and resource allocation in telecommunication systems, modeling of communication networks, analysis of cognitive radio networks, modeling and analysis of wireless sensor networks, developing efficient decoding algorithms for LDPC codes, channel modeling, traffic estimation for the Internet, and cross layer analysis. Dr. Alfa also works in the application of queueing theory to other areas such as transportation systems, manufacturing systems and healthcare systems. He has authored two books, "Queueing Theory for Telecommunications: Discrete Time Modelling of a Single Node System”, published by Springer in 2010, and "Applied Discrete-Time Queue" published in 2015 also by Springer. 